\title{
Research Article \\ On the Boundary Value Condition of an Isotropic Parabolic Equation
}

\author{
Qitong Ou (i) and Huashui Zhan \\ School of Applied Mathematics, Xiamen University of Technology, Xiamen 361024, China \\ Correspondence should be addressed to Qitong Ou; ouqitong@xmut.edu.cn
}

Received 12 June 2020; Revised 19 September 2020; Accepted 4 October 2020; Published 27 November 2020

Academic Editor: Wilfredo Urbina

Copyright () 2020 Qitong Ou and Huashui Zhan. This is an open access article distributed under the Creative Commons Attribution License, which permits unrestricted use, distribution, and reproduction in any medium, provided the original work is properly cited.

The well-posedness problem of anisotropic parabolic equation with variable exponents is studied in this paper. The weak solutions and the strong solutions are introduced, respectively. By a generalized Gronwall inequality, the stability of strong solutions to this equation is established, and the uniqueness of weak solutions is proved. Compared with the related works, a new boundary value condition, $\prod_{i=1}^{N} a_{i}(x, t)=0,(x, t) \in \partial \Omega \times[0, T]$, is introduced the first time and has been proved that it can take place of the Dirichlet boundary value condition in some way.

\section{Introduction}

In this paper, we mainly pay attention on the stability of solutions to the following anisotropic parabolic equation with variable exponents:

$$
\begin{aligned}
u_{t}= & \sum_{i=1}^{N} \frac{\partial}{\partial x_{i}}\left(a_{i}(x, t)\left|u_{x_{i}}\right|^{p_{i}(x, t)-2} u_{x_{i}}\right) \\
& +\sum_{i=1}^{N} \frac{\partial b_{i}(u, x, t)}{\partial x_{i}}, \quad(x, t) \in Q_{T},
\end{aligned}
$$

with the initial value

$$
u(x, 0)=u_{0}(x), \quad x \in \Omega
$$

and the boundary value condition

$$
u(x, t)=0, \quad(x, t) \in \partial \Omega \times(0, T) .
$$

Here, $a_{i}(x, t) \geq 0, p_{i}(x, t)>1, a_{i}(x, t) \in C\left(\overline{Q_{T}}\right), p_{i}(x, t) \epsilon$ $C\left(\bar{Q}_{T}\right)$, and $\Omega \subset \mathbb{R}^{N}$ is a bounded domain with the smooth boundary $\partial \Omega, Q_{T}=\Omega \times(0, T)$.

When $p_{i}(x, t)=p$ is a constant, $i=1,2, \cdots, N$, equation (1) arises in the mathematical modelling of various physical processes such as flows of incompressible turbulent fluids, gases in pipes, and processes of filtration in glaciology. The equation in this case has been studied widely [1-5]. When $p_{i}(x, t)=p(x, t)$ is a measurable function, $i=1,2, \cdots, N$, equation (1) is similar with the equation with the type

$$
u_{t}=\operatorname{div}\left(a(x, t)|\nabla u|^{p(x, t)} \nabla u\right)+f(x, t, u, \nabla u)
$$

which arises in the phenomena of electrorheological fluids [6, 7]. The existence of solutions of the initial-boundary value problem to this equation can be found in [8-11]. Also, one can refer to [12-18] for some other related works.

$$
\text { If } a_{i}(x, t)=a_{i}(x) \text { and satisfies }
$$

$$
\left.a_{i}(x)\right|_{x \in \Omega}>0,\left.a_{i}(x)\right|_{x \in \partial \Omega}=0, \quad i=1,2, \cdots, N,
$$

the well-posedness problem of the following equations

$$
u_{t}=\sum_{i=1}^{N} \frac{\partial}{\partial x_{i}}\left(a_{i}(x)\left|u_{x_{i}}\right|^{p_{i}(x)-2} u_{x_{i}}\right)+f(x, t, u, \nabla u)
$$

has been studied by the second author in recent years [1921 ]. Instead of boundary value condition (3), only a partial boundary value condition 


$$
u(x, t)=0, \quad(x, t) \in \sum_{p} \times(0, T)
$$

is imposed, where $\sum_{p} \in \partial \Omega$ is a relatively open subset which has different expression according to different kinds of $f(x, t, u, \nabla u)$ and sometimes is just an empty set [19-21].

Compared with $[19,20]$ and [21], since the diffusion coefficient $a_{i}(x, t)$ and the variable exponent $p_{i}(x, t)$ both depend on the time variable $t$, equation (1) has a wider applications than equation (6), and in mathematical theory, there are some essential difficulties to be overcome. More than that, instead of (5), we only assume that

$$
\begin{aligned}
& a_{i}(x, t)>0, \quad(x, t) \in \Omega \times[0, T], i=1,2, \cdots, N, \\
& \prod_{i=1}^{N} a_{i}(x, t)=0, \quad(x, t) \in \partial \Omega \times[0, T]
\end{aligned}
$$

and do not require that

$$
a_{i}(x, t)=0, \quad(x, t) \in \partial \Omega \times[0, T], i=1,2, \cdots, N,
$$

which is similar as (5) in [19-21].

To see the essential difference between (9) and (10), let us give a special case of equation when $N=2, \Omega \subset \mathbb{R}^{2}, \partial \Omega=\bar{\Gamma}_{1}$ $\cup \bar{\Gamma}_{2}$, and $\Gamma_{1}$ and $\Gamma_{2}$ are relatively open subset of $\partial \Omega, \Gamma_{1} \cap$ $\Gamma_{2}=\varnothing$. Consider the equation

$$
\begin{aligned}
u_{t}= & \frac{\partial}{\partial x_{1}}\left(a_{1}(x)\left|u_{x_{1}}\right|^{p_{1}(x)-2} u_{x_{i}}\right) \\
& +\frac{\partial}{\partial x_{2}}\left(a_{2}(x)\left|u_{x_{1}}\right|^{p_{2}(x)-2} u_{x_{i}}\right), \quad(x, t) \in Q_{T},
\end{aligned}
$$

where

$$
\begin{aligned}
& a_{1}(x)=0, \quad x \in \Gamma_{1}, a_{1}(x)>0, x \in \Gamma_{2}, \\
& a_{2}(x)>0, \quad x \in \Gamma_{2}, a_{2}(x)=0, x \in \Gamma_{2},
\end{aligned}
$$

then (9) is true, i.e.,

$$
\prod_{i=1}^{2} a_{i}(x)=0, \quad(x, t) \in \partial \Omega \times[0, T]
$$

More precisely, for example,

$$
\begin{aligned}
\Omega & =\left\{x=\left(x_{1}, x_{2}\right): 1<x_{1}^{2}+x_{2}^{2}<4\right\}, \\
a_{1}(x) & =x_{1}^{2}+x_{2}^{2}-1, \\
a_{2}(x) & =4-\left(x_{1}^{2}+x_{2}^{2}\right) .
\end{aligned}
$$

However, in (12) and (13),

$$
a_{1}(x)+a_{2}(x)>0, \quad x \in \partial \Omega \text {. }
$$

This fact makes us feel that only under the boundary value condition (3), the uniqueness (or the stability) of weak solutions to equation (11) can be true. The following works seem to supply more evidences. One is [22] in which the equation

$$
\begin{aligned}
v_{t}= & \operatorname{div}\left(\left|\nabla v^{m}\right|^{p(x, t)-2} \nabla v^{m}+b(x, t) \nabla v^{m}\right) \\
& +v^{q(x, t)}, \quad(x, t) \in Q_{T}
\end{aligned}
$$

is studied. The others are the equations arising from the double phase obstacle problems

$$
\begin{aligned}
v_{t}= & \operatorname{div}\left(a(x)|\nabla v|^{p-2} \nabla v+b(x)|\nabla v|^{q-2} \nabla v\right) \\
& +f(x, t, v, \nabla v), \quad(x, t) \in Q_{T},
\end{aligned}
$$

where $a(x)+b(x)>0$, which have gained a wide attention in recent years, one can refer to $[23,24]$ and the references therein. In these papers, the boundary value condition (3) is imposed without exception.

The main dedication of this paper is that the stability of weak solutions to equation (11)(in general, (1)) can be established independent of boundary value condition (3). Such a conclusion totally overthrows our imagination. In theory, condition (9) can take place of boundary value condition (3) is found the first time. In applications, condition (9) reflects a synthesized effect of an anisotropic diffusion process.

This paper is arranged as follows. In Section 1, we have given a simple introduction. In Section 2, we will introduce the definitions of weak solution and strong weak solution, respectively, quote some basic lemmas, and give the main results. In Section 3, we will study the stability of weak solutions to equation (1) with the new boundary value condition (9). In Section 4, we will study the uniqueness of weak solution to equation (1) independent of the boundary value condition (7). In Section 5, we will give the outline of the proof on the existence of strong solutions.

\section{Definitions and Main Results}

We denote

$$
\begin{aligned}
& p_{i}^{+}=\max _{(x, t) \in Q_{T}} p_{i}(x, t), p_{i t}^{+}=\max _{x \in \bar{\Omega}} p_{i}(x, t), \quad i=1,2, \cdots, N, \\
& p_{i}^{-}=\min _{(x, t) \in Q_{T}} p_{i}(x, t), p_{i t}^{-}=\min _{x \in \bar{\Omega}} p_{i}(x, t), \quad i=1,2, \cdots, N, \\
& p_{-}=\min \left\{p_{1}^{-}, p_{2}^{-}, \cdots, p_{N-1}^{-}, p_{N}^{-}\right\}, \quad p_{-}>1, \\
& p_{+}=\max \left\{p_{1}^{+}, p_{2}^{+}, \cdots, p_{N-1}^{+}, p_{N}^{+}\right\} .
\end{aligned}
$$

First of all, let us introduce the definition of solutions. 
Definition 1. A function $u(x, t)$ is said to be a weak solution of equation (1), if

$$
\begin{gathered}
u \in L^{\infty}\left(Q_{T}\right), u_{t} \in L^{p_{+}{ }^{\prime}}\left(0, T ; W^{-1, p_{+}{ }^{\prime}}(\Omega)\right) a_{i}(x, t) \\
\cdot\left|u_{x_{i}}\right|^{p_{i}(x, t)} \in L^{1}\left(Q_{T}\right), \quad i=1,2, \cdots, N
\end{gathered}
$$

and for any function $\varphi \in C_{0}^{1}\left(Q_{T}\right)$,

$$
\begin{aligned}
\iint_{Q_{T}} u_{t} \varphi d x d t & +\sum_{i=1}^{N} \iint_{Q_{T}}\left[a_{i}(x, t)\left|u_{x_{i}}\right|^{p_{i}(x, t)-2} u_{x_{i}} \cdot \varphi_{x_{i}}\right. \\
& \left.+b_{i}(u, x, t) \cdot \varphi_{x_{i}}\right] d x d t=0 .
\end{aligned}
$$

This definition of weak solution is similar as that defined in [20], where $a_{i}(x, t)=a_{i}(x), p_{i}(x, t)=p_{i}$ is a constant. Also, we can prove the existence of weak solutions similar as that defined in [20], so we do not repeat the details in this paper. As an improvement from the existing result in [20], we introduce the following definition.

Definition 2. A function $u(x, t)$ is said to be a strong solution of equation (1) with the initial value (2), if

$u \in L^{\infty}\left(Q_{T}\right), u_{t} \in L^{2}\left(Q_{T}\right), a_{i}(x, t)\left|u_{x_{i}}\right|^{p_{i}(x, t)} \in L^{1}\left(Q_{T}\right), \quad i=1,2, \cdots, N$,

and for any function $\varphi \in C_{0}^{1}\left(Q_{T}\right)$, $u$ satisfies $(21)$ and the initial value is satisfied in the sense

$$
\lim _{t \rightarrow 0} \int_{\Omega}\left(u(x, t)-u_{0}(x)\right) \phi(x) d x=0, \quad \phi(x) \in C_{0}^{\infty}(\Omega) .
$$

The proof of the existence of strong solution will be given at Section 5 of this paper. Since $a_{i}(x, t)$ is positive when $x \in \Omega$, (22) means that $u_{t}$ and $\nabla u$ exist almost everywhere in $Q_{T}$. This is the reason that we call $u(x, t)$ as a strong solution of equation (1). Moreover, from Definition 2, for all $\varphi(x, t) \epsilon$ $L^{p_{+}}\left(0, T ; W_{0}^{1, p_{+}}(\Omega)\right)$, we still have the integral equality $(21)$, which implies that

$$
\left\|u_{t}\right\|_{L^{p_{+}}\left(0, T ; W^{\left.-1, p_{+}{ }^{\prime}(\Omega)\right)}\right.} \leq c .
$$

Thus, if $u(x, t)$ is a strong solution of equation (1), then it is a weak solution.

Secondly, in order to make the paper sufficiently selfcontained and present our discussions in a straightforward manner, let us briefly recall some preliminary results on properties of variable exponent Lebesgue spaces $L^{p(x)}(\Omega)$ and variable exponent Sobolev spaces $W^{1, p(x)}(\Omega)$ [24-26].

Set

$$
C_{+}(\bar{\Omega})=\left\{h \in C(\bar{\Omega}): \min _{x \in \bar{\Omega}} h(x)>1\right\} .
$$

For any $h \in C_{+}(\bar{\Omega})$, we define

$$
\begin{aligned}
& h^{+}=\sup _{x \in \Omega} h(x), \\
& h^{-}=\inf _{x \in \Omega} h(x) .
\end{aligned}
$$

For any $p \in C_{+}(\bar{\Omega})$, let $L^{p(x)}(\Omega)$ consist of all measurable real-valued functions $u(x)$ satisfying

$$
\int_{\Omega}|u(x)|^{p(x)} d x<\infty
$$

endowed with the Luxemburg norm

$$
\|u\|_{L^{p(x)}(\Omega)}=\inf \left\{\lambda>0: \int_{\Omega}\left|\frac{u(x)}{\lambda}\right|^{p(x)} d x \leq 1\right\} .
$$

Define

$$
W^{1, p(x)}(\Omega)=\left\{u \in L^{p(x)}(\Omega):|\nabla u| \in L^{p(x)}(\Omega)\right\},
$$

endowed with the norm

$$
\|u\|_{W^{1, p(x)}(\Omega)}=\|u\|_{L^{p(x)}(\Omega)}+\|\nabla u\|_{L^{p(x)}(\Omega)} .
$$

Let $W_{0}^{1, p(x)}(\Omega)$ be the closure space of $C_{0}^{\infty}(\Omega)$ in $W^{1, p(x)}(\Omega)$.

From [25-27], we have

Lemma 3. The following three statements are true.

(i) The space $\left(L^{p(x)}(\Omega),\|\cdot\|_{L^{p(x)}(\Omega)}\right), \quad\left(W^{1, p(x)}(\Omega), \| \cdot\right.$ $\left.\|_{W^{1, p(x)}(\Omega)}\right)$, and $W_{0}^{1, p(x)}(\Omega)$ are reflexive Banach spaces

(ii) ( $p(x)$-Hölder's inequality), let $q_{1}(x)$ and $q_{2}(x)$ be real functions satisfying $1 / q_{1}(x)+1 / q_{2}(x)=1$ with $q_{1}(x)$ $>1$. Then, the conjugate space of $L^{q_{1}(x)}(\Omega)$ is $L^{q_{2}(x)}$ $(\Omega)$. For any $u \in L^{q_{1}(x)}(\Omega)$ and $v \in L^{q_{2}(x)}(\Omega)$, we have

$$
\left|\int_{\Omega} u v d x\right| \leq 2\|u\|_{L^{q_{1}(x)}(\Omega)}\|v\|_{L^{q_{2}(x)}(\Omega)}
$$

(iii) There are the following properties:

(1) if $\|u\|_{L^{p(x)}(\Omega)}=1$, then $\int_{\Omega}\|u\|^{p(x)} d x=1$

(2) if $\|u\|_{L^{p(x)}(\Omega)}>1$, then $\|u\|_{L^{p(x)}(\Omega)}^{p^{-}} \leq \int_{\Omega}|u|^{p(x)} d x \leq$ $\|u\|_{L^{p(x)}(\Omega)}^{p^{+}}$

(3) if $\|u\|_{L^{p(x)}(\Omega)}<1$, then $\|u\|_{L^{p(x)}(\Omega)}^{p^{+}} \leq \int_{\Omega}|u|^{p(x)} d x \leq$ $\|u\|_{L^{p(x)}(\Omega)}^{p^{-}}$ 
Basing on Lemma 3, by generalizing the Gronwall inequality, we will prove the following stability theorems, in which the initial values satisfy

$$
\begin{array}{ll}
u_{0} \in L^{\infty}(\Omega),\left|u_{0 x_{i}}\right| \in L^{p_{i}}(\Omega), & i=1,2, \cdots, N, \\
v_{0} \in L^{\infty}(\Omega),\left|v_{0 x_{i}}\right| \in L^{p_{i}}(\Omega), & i=1,2, \cdots, N .
\end{array}
$$

Theorem 4. Let $u(x, t)$ and $v(x, t)$ be two strong solutions of (1) with the initial values $u_{0}(x)$ and $v_{0}(x)$, respectively; $p_{-}>$ 1 and $a_{i}(x, t) \in C\left(\bar{Q}_{T}\right)$ satisfy (8) and (9) and

$$
\int_{\Omega} a_{i}(x, t)^{-1 /\left(p_{i}(x, t)-1\right)} d x \leq c(T), \quad i=1,2, \cdots, N,
$$

$b_{i}(s, x, t)$ satisfies

$\left|b_{i}(u, x, t)-b_{i}(v, x, t)\right| \leq c a_{i}(x, t)^{1 / p_{i}(x, t)}|u-v|, \quad i=1,2, \cdots, N$.

If for $\eta$ small enough,

$$
\begin{aligned}
& \frac{1}{\eta}\left(\int_{\Omega \backslash \Omega_{\eta t}} a_{i}(x, t)\left|\left(\prod_{j=1}^{N} a_{j}(x)\right)_{x_{i}}\right|^{p_{i}(x, t)} d x\right)^{1 / p_{i t}^{+}} \\
& \quad \leq c(T), \quad i=1,2, \cdots, N,
\end{aligned}
$$

then

$$
\int_{\Omega}|u(x, t)-v(x, t)| d x \leq \int_{\Omega}\left|u_{0}(x)-v_{0}(x)\right| d x, \quad t \in[0, T),
$$

where for any $t \in[0, T), \Omega_{\eta t}=\left\{x \in \Omega:\left(\prod_{j=1}^{N} a_{j}(x, t)\right)>\eta\right\}$.

In this paper, the constant $c(T)$ represents that $c$ depends on $T$. If we only want to prove the uniqueness of weak solutions, condition (34) is not necessary; we have the following result.

Theorem 5. Let $p_{-}>1, a_{i}(x, t) \in C^{1}\left(\bar{Q}_{T}\right)$ satisfy (8) and (9) and $\left(\partial a_{i}(x, t)\right) / \partial t \leq 0, b_{i}(s, x, t)$ be a Lipschitz function on $\mathbb{R}$ $\times \bar{\Omega} \times[0, T]$. If $u(x, t)$ and $v(x, t)$ are two strong solutions of equation (1) with the initial values $u_{0}(x)$ and $v_{0}(x)$, respectively, then for any $\Omega_{1} \subset \subset \Omega$,

$$
\int_{\Omega_{1}}|u(x, t)-v(x, t)|^{2} d x \leq c\left(\Omega_{1}\right) \int_{\Omega}\left|u_{0}(x)-v_{0}(x)\right|^{2} d x
$$

which implies that the uniqueness of weak solution is true.

One can see that both Theorem 4 and Theorem 5 imply the uniqueness of solution is true. However, in Theorem 5, the convection function $b_{i}(\cdot, x, t)$ is independent of the diffusion coefficient $a_{i}(x, t)$, so as a uniqueness theorem, it is a better than Theorem 4.

\section{The Stability of Strong Solutions Independent of the Boundary Value Condition}

For small $\eta>0$, let

$$
\begin{aligned}
& h_{\eta}(s)=\frac{2}{\eta}\left(1-\frac{|s|}{\eta}\right)_{+}, \\
& S_{\eta}(s)=\int_{0}^{s} h_{\eta}(\tau) d \tau .
\end{aligned}
$$

Obviously, $h_{\eta}(s) \in C(\mathbb{R})$, and

$$
\left|S_{\eta}(s)\right| \leq 1 ; \lim _{\eta \rightarrow 0} S_{\eta}(s)=\operatorname{sgn} s, \lim _{\eta \rightarrow 0} s S_{\eta}^{\prime}(s)=0 .
$$

In addition, if we denote $H_{\eta}(s)=\int_{0}^{s} S_{\eta}(\tau) d \tau$, then

$$
\lim _{\eta \rightarrow 0} H_{\eta}(s)=|s|, s \in(-\infty,+\infty) .
$$

At first, we give a generalization of the Gronwall inequality.

Lemma 6. If $a(x, t)|u(x, t)|^{p(x, t)}, a(x, t)|v(x, t)|^{p(x, t)} \in L^{1}\left(Q_{T}\right)$ and

$$
\begin{aligned}
& \int_{\Omega} a(x, s)|u(x, s)-v(x, s)|^{p(x, s)} d x \\
& \leq \int_{\Omega} a(x, \tau)|u(x, \tau)-v(x, \tau)|^{p(x, \tau)} d x \\
& \quad+c\left(\int_{\tau}^{s} \int_{\Omega} a(x, t)|u-v|^{p(x, t)} d x d t\right)^{l},
\end{aligned}
$$

where $0<l \leq 1$, then,

$$
\begin{aligned}
& \int_{\Omega} a(x, t)|u(x, s)-v(x, s)|^{p(x, t)} d x \\
& \quad \leq c \int_{\Omega} a(x, \tau)|u(x, \tau)-v(x, \tau)|^{p(x, t)} d x .
\end{aligned}
$$

Proof. Define that $\kappa(t)=\int_{\Omega} a(x, t)|u(x, t)-v(x, t)|^{p(x, t)} d x$. Without loss of the generality, we may assume that there exists $\tau \in[0, T), \kappa(\tau)>0$. Then, for any $s>\tau, \int_{\tau}^{s} k(t) d t>0$. Denoting

$$
\begin{aligned}
\tau_{0} & =\max \{t \in[\tau, s], \kappa(t)>0\}, \\
\int_{\tau}^{\tau_{0}} k(t) d t & =c_{1},
\end{aligned}
$$

then, $\tau<\tau_{0} \leq s$, and

$$
\int_{\tau}^{s} k(t) d t \geq \int_{\tau}^{\tau_{0}} k(t) d t=c_{1}
$$


Since $\quad a(x, t)|u(x, t)|^{p(x, t)}, a(x, t)|v(x, t)|^{p(x, t)} \in L^{1}\left(Q_{T}\right)$, there exists a constant $C>0$ such that

$$
\frac{c\left(\int_{\tau}^{s} k(t) d t\right)^{l}}{\int_{\tau}^{s} k(t) d t} \leq \frac{c\left(\int_{\tau}^{s} k(t) d t\right)^{l}}{c_{1}} \leq C=C\left(c, c_{1}, T\right) .
$$

Combing (41) with (45), we obtain

$$
\kappa(s)-\kappa(\tau) \leq(C+c) \int_{\tau}^{s} k(t) d t .
$$

Using the Gronwall inequality, we have

$$
\begin{aligned}
& \int_{\Omega} a(x, s)|u(x, s)-v(x, s)|^{p(x, s)} d x \\
& \quad \leq c \int_{\Omega} a(x, \tau)|u(x, \tau)-v(x, \tau)|^{p(x, \tau)} d x .
\end{aligned}
$$

If $u(x, t), v(x, t) \in L^{\infty}\left(Q_{T}\right)$, this lemma has been proved in [19] recently.

Secondly, we give the proof of Theorem 4.

Proof of Theorem 4. Let $u(x, t)$ and $v(x, t)$ be two strong solutions of equation (1) with the initial values $u_{0}(x)$ and $v_{0}(x)$, respectively.

For any $t \in[0, T]$, set $\Omega_{\eta t}=\left\{x \in \Omega: \prod_{i=1}^{N} a_{i}(x, t)>\eta\right\}$, and

$$
\phi_{\eta t}(x)=\left\{\begin{array}{l}
1, \quad \text { if } x \in \Omega_{\eta t}, \\
\frac{1}{\eta} \prod_{i=1}^{N} a_{i}(x, t), \quad \text { if } x \in \Omega \backslash \Omega_{\eta t} .
\end{array}\right.
$$

By a process of limit, we can choose $\chi_{[\tau, s} \phi_{\eta t}(x) S_{\eta}(u-v)$ as the test function. Here, $\chi_{[\tau, s]}$ is the characteristic function of $[\tau, s] \subset(0, T)$. Then,

$$
\begin{aligned}
& \int_{\tau}^{s} \int_{\Omega} \phi_{\eta t} S_{\eta}(u-v) \frac{\partial(u-v)}{\partial t} d x d t+\sum_{i=1}^{N} \int_{\tau}^{s} \int_{\Omega} a_{i}(x, t) \\
& \cdot\left(\left|u_{x_{i}}\right|^{p_{i}(x, t)-2} u_{x_{i}}-\left|v_{x_{i}}\right|^{p_{i}(x, t)-2} \nabla v\right)\left(u_{x_{i}}-v_{x_{i}}\right) h_{\eta} \\
& \cdot(u-v) \phi_{\eta t}(x) d x d t+\sum_{i=1}^{N} \int_{\tau}^{s} \int_{\Omega} a_{i}(x, t)\left(\left|u_{x_{i}}\right| p_{i}(x, t)-2 u_{x_{i}}\right. \\
& \left.\quad-\left|v_{x_{i}}\right|^{p_{i}(x, t)-2} v_{x_{i}}\right)(u-v) S_{\eta}(u-v) \frac{\partial \phi_{\eta t}}{\partial x_{i}} d x d t \\
& +\sum_{i=1}^{N} \int_{\tau}^{s} \int_{\Omega}\left[b_{i}(u, x, t)-b_{i}(v, x, t)\right] \frac{\partial \phi_{\eta t}}{\partial x_{i}} S_{\eta}(u-v) d x d t \\
& \quad+\sum_{i=1}^{N} \int_{\tau}^{s} \int_{\Omega}\left[b_{i}(u, x, t)-b_{i}(v, x, t)\right] \\
& \cdot(u-v)_{x_{i}} \phi_{\eta t} h_{\eta}(u-v) d x d t=0 .
\end{aligned}
$$

In the first place, using the dominated convergence theorem, we have

$$
\begin{aligned}
& \lim _{\eta \rightarrow 0} \int_{\tau}^{s} \int_{\Omega} \phi_{\eta t}(x) S_{\eta}(u-v) \frac{\partial(u-v)}{\partial t} d x d t \\
& \quad=\int_{\tau}^{s} \int_{\Omega} \operatorname{sgn}(u-v) \frac{\partial(u-v)}{\partial t} d x d t \\
& \quad=\lim _{\eta \rightarrow 0} \int_{\tau}^{s} \int_{\Omega} \frac{\partial H_{\eta}(u-v)}{\partial t} d x d t \\
& \quad=\lim _{\eta \rightarrow 0} \int_{\Omega}\left[H_{\eta}(u-v)(x, s)-H_{\eta}(u-v)(x, \tau)\right] d x \\
& \quad=\int_{\Omega}|u-v|(x, s) d x-\int_{\Omega}|u-v|(x, \tau) d x,
\end{aligned}
$$

and for any $1 \leq i \leq N$,

$$
\begin{gathered}
\int_{\Omega} a_{i}(x, t)\left(\left|u_{x_{i}}\right|^{p_{i}(x, t)-2} u_{x_{i}}-\left|v_{x_{i}}\right|^{p_{i}(x, t)-2} v_{x_{i}}\right) \\
\cdot\left(u_{x_{i}}-v_{x_{i}}\right) h_{\eta}(u-v) \phi_{\eta t}(x) d x \geq 0 .
\end{gathered}
$$

In the second place, we notice $\partial \phi_{\eta t} / \partial x_{i}=(1 / \eta)$ $\left(\prod_{j=1}^{N} a_{j}(x, t)\right)_{x_{i}}$ when $x \in \Omega \backslash \Omega_{\eta t}$; in the other places, it is identical to zero. Since we assume that

$$
\prod_{j=1}^{N} a_{j}(x, t)=0, \quad(x, t) \in \partial \Omega \times[0, T]
$$

by Lemma 3, we have

$$
\begin{aligned}
& \left|\int_{\Omega} a_{i}(x, t)\left(\left|u_{x_{i}}\right|^{p_{i}(x, t)-2} u_{x_{i}}-\left|v_{x_{i}}\right|^{p_{i}(x, t)-2} v_{x_{i}}\right) \frac{\partial \phi_{\eta t}}{\partial x_{i}} S_{\eta}(u-v) d x\right| \\
& =\left|\int_{\Omega \mid \Omega_{\eta t}} a_{i}(x, t)\left(\left|u_{x_{i}}\right|_{i}^{p_{i}(x, t)-2} u_{x_{i}}-\left|v_{x_{i}}\right|^{p_{i}(x, t)-2} v_{x_{i}}\right) \frac{\partial \phi_{\eta t}}{\partial x_{i}} S_{\eta}(u-v) d x\right| \\
& \leq \frac{1}{\eta} \int_{\Omega \mid \Omega_{\eta^{t}}} a_{i}(x, t)\left(\left|u_{x_{i}}\right|^{p_{i}(x, t)-1}+\left|v_{x_{i}}\right|^{p_{i}(x, t)-1}\right)\left(\prod_{j=1}^{N} a_{j}(x, t)\right)_{x_{i}} S_{\eta}(u-v) \mid d x \\
& \leq c \frac{1}{\eta}\left(\int_{\Omega \mid \Omega_{\eta t}}\left(a_{i}(x, t)\left|u_{x_{i}}\right|^{p_{i}(x, t)}+\left|v_{x_{i}}\right|^{p_{i}(x, t)}\right) d x\right)^{1 / p_{i t}^{\prime}+} \\
& \cdot\left(\int_{\Omega \mid \Omega_{n t}} a_{i}(x, t)\left|\left(\prod_{j=1}^{N} a_{j}(x, t)\right)_{x_{i}}\right|^{p_{i}(x, t)} d x\right)^{1 / p_{i t}^{+}} \\
& \leq c\left[\left(\int_{\Omega \mid \Omega_{\eta t}} a_{i}(x, t)\left|u_{x_{i}}\right|^{p_{i}(x, t)} d x\right)^{\left(p_{i}-1\right) / p_{i}}+\left(\int_{\Omega \mid \Omega_{n t}} a_{i}(x)\left|v_{x_{i}}\right|^{p_{i}(x, t)} d x\right)^{1 / p_{i t}^{\prime}+}\right] \\
& \cdot\left[\frac{1}{\eta}\left(\int_{\Omega \mid \Omega_{n t}} a_{i}(x, t)\left|\left(\prod_{j=1}^{N} a_{j}(x, t)\right)_{x_{i}}\right|^{p_{i t}} d x\right)^{1 / p_{t i t}^{*}}\right] \\
& \leq c\left[\left(\int_{\Omega \mid \Omega_{\eta t}} a_{i}(x, t)\left|u_{x_{i}}\right|^{p_{i}(x, t)} d x\right)^{1 / p^{\prime}{ }^{\prime}+}+\left(\int_{\Omega \mid \Omega_{\eta t}} a_{i}(x, t)\left|v_{x_{i}}\right|^{p_{i t}} d x\right)^{1 / p_{i t}^{\prime}+}\right] \text {, }
\end{aligned}
$$

where $p_{i t}^{+}=\max _{x \in \bar{\Omega}} p(x, t)$ and $p_{i}^{\prime}(x, t)=p(x, t) /(p(x, t)-1)$. 
(53) implies

$$
\begin{gathered}
\lim _{\eta \rightarrow 0}\left|\int_{\tau}^{s} \int_{\Omega} a_{i}(x, t)\left(\left|u_{x_{i}}\right|^{p_{i}(x, t)-2} u_{x_{i}}-\left|v_{x_{i}}\right|^{p_{i}(x, t)-2} v_{x_{i}}\right) \frac{\partial \phi_{\eta t}}{\partial x_{i}} S_{\eta}(u-v) d x d t\right| \\
\leq \operatorname{cim}_{\eta \rightarrow 0} \int_{\tau}^{s}\left[\left(\int_{\Omega \backslash \Omega_{\eta t}} a_{i}(x, t)\left|u_{x_{i}}\right|^{p_{i}(x, t)} d x\right)^{1 / p^{\prime}{ }_{i t}^{+}}\right. \\
\left.\quad+\left(\int_{\Omega \backslash \Omega_{\eta t}} a_{i}(x, t)\left|v_{x_{i}}\right|^{p_{i}(x, t)} d x\right)^{1 / p^{\prime}{ }_{i t}^{+}}\right] d t=0 .
\end{gathered}
$$

In the third place, since $a_{i}(x, t)$ satisfies condition (34), we can deduce that

$\lim _{\eta \rightarrow 0} \sum_{i=1}^{N} \int_{\tau}^{s} \int_{\Omega}\left[b_{i}(u, x, t)-b_{i}(v, x, t)\right](u-v)_{x_{i}} \phi_{\eta} h_{\eta}(u-v) d x d t=0$.

In details,

$$
\begin{aligned}
& \left|\int_{\Omega}\left[b_{i}(u, x, t)-b_{i}(v, x, t)\right](u-v)_{x_{i}} h_{\eta}(u-v) d x\right| \\
& =\left|\int_{\{\Omega:|u-v|<\eta\}}\left[b_{i}(u, x, t)-b_{i}(v, x, t)\right](u-v)_{x_{i}} h_{\eta}(u-v) d x\right| \\
& \quad \leq \int_{\{\Omega:|u-v|<\eta\}}\left|a_{i}(x, t)^{1 / p_{i}(x, t)}(u-v)_{x_{i}}\right| a_{i}(x, t)^{-1 / p_{i}(x, t)} \\
& \quad \cdot\left|(u-v) h_{\eta}(u-v)\right| d x .
\end{aligned}
$$

If the set $\{\Omega:|u-v|=0\}$ has a positive measure, by condition (34), we have

$$
\begin{aligned}
\lim _{\eta \rightarrow 0}\left|\int_{\{\Omega:|u-v|<\eta\}}\left[b_{i}(u, x, t)-b_{i}(v, x, t)\right](u-v)_{x_{i}} h_{\eta}(u-v) d x\right| \\
\leq c\left(\int_{\{\Omega:|u-v|=0\}}\left(a_{i}(x, t)^{1 / p_{i}(x, t)}\left|u_{x_{i}}-v_{x_{i}}\right|\right)^{p_{i}(x, t)} d x\right)^{1 / p_{i t}^{+}} \\
\quad \cdot\left(\int_{\Omega} a_{i}(x, t)^{-1 /\left(p_{i}(x, t)-1\right)} d x\right)^{1 / p^{\prime}{ }_{1 i t}}=0
\end{aligned}
$$

where $p_{1 i t}^{\prime}=p_{i t}^{\prime+}$ or $p_{i t}^{\prime-}$ according to $\int_{\Omega} a_{i}(x, t)^{-1 /\left(p_{i}(x, t)-1\right)} d x$ $\leq 1$ or $\int_{\Omega} a_{i}(x, t)^{-1 /\left(p_{i}(x, t)-1\right)} d x>1$ from Lemma 3 .

If the set $\{\Omega:|u-v|=0\}$ only has zero measure, since $a_{i}(x, t)^{-1 /\left(p_{i}(x, t)-1\right)} \in L^{1}(\Omega)$,

$$
\begin{aligned}
\lim _{\eta \rightarrow 0}\left|\int_{\{\Omega:|u-v|<\eta\}}\left[b_{i}(u, x, t)-b_{i}(v, x, t)\right](u-v)_{x_{i}} h_{\eta}(u-v) d x\right| \\
\leq c\left(\int_{\Omega}\left(a_{i}^{1 / p_{i}}\left|u_{x_{i}}-v_{x_{i}}\right|\right)^{p_{i}} d x\right)^{1 / p_{i}} \\
\quad \cdot\left(\int_{\{\Omega:|u-v|=0\}} a_{i}(x, t)^{-1 /\left(p_{i}-1\right)} d x\right)^{\left(p_{i}-1\right) / p_{i}} \\
\leq c\left(\int_{\Omega} a_{i}(x)\left(\left|u_{x_{i}}\right| p_{i}+\left|v_{x_{i}}\right| p_{i}\right) d x\right)^{1 / p_{i}} \\
\quad\left(\int_{\{\Omega:|u-v|=0\}} a_{i}(x, t)^{-1 /\left(p_{i}-1\right)} d x\right)^{\left(p_{i}-1\right) / p_{i}}=0 .
\end{aligned}
$$

Finally, by condition (34), we have

$$
\begin{aligned}
& \lim _{\eta \rightarrow 0}\left|\int_{\Omega}\left[b_{i}(u, x, t)-b_{i}(v, x, t)\right] \frac{\partial \phi_{\eta t}}{\partial x_{i}} S_{\eta}(u-v) d x\right| \\
& =\lim _{\eta \rightarrow 0}\left|\int_{\Omega \backslash \Omega_{\eta t}}\left[b_{i}(u, x, t)-b_{i}(v, x, t)\right] \frac{\partial \phi_{\eta t}}{\partial x_{i}} S_{\eta}(u-v) d x\right| \\
& \leq \lim _{\eta \rightarrow 0} \frac{1}{\eta} \int_{\Omega \backslash \Omega_{\eta t}} a_{i}^{1 / p_{i}(x, t)}\left|\left(\prod_{j=1}^{N} a_{j}(x, t)\right)_{x_{i}}\right|\left|S_{\eta}(u-v)(u-v)\right| d x \\
& \leq \lim _{\eta \rightarrow 0} \frac{1}{\eta}\left(\int_{\Omega \backslash \Omega_{\eta t}} a_{i}(x, t)\left|\left(\prod_{j=1}^{N} a_{j}(x, t)\right)_{x_{i}}\right|^{p_{i}(x, t)} d x\right)^{1 / p_{i t}^{+}} \\
& \cdot\left(\int_{\Omega \backslash \Omega_{\eta t}}\left|S_{\eta}(u-v)(u-v)\right|^{p_{i}(x, t) /\left(p_{i}(x, t)-1\right)} d x\right)^{1 / p_{i t}^{\prime}+} \\
& \leq \lim _{\eta \rightarrow 0}\left(\int_{\Omega \backslash \Omega_{\eta t}}\left|S_{\eta}(u-v)(u-v)\right|^{p_{i}(x, t) /\left(p_{i}(x, t)-1\right)} d x\right)^{1 / p_{i t}^{\prime}+} \\
& \leq c\left(\int_{\Omega}|u-v| d x\right)^{1 / p_{i t}^{\prime}+}
\end{aligned}
$$

since $u(x, t), v(x, t) \in L^{\infty}\left(Q_{T}\right)$.

Now, let $\eta \rightarrow 0$ in (42). We easily obtain that

$$
\begin{aligned}
\int_{\Omega}|u(x, s)-v(x, s)| d x \leq & \int_{\Omega}|u(x, \tau)-v(x, \tau)| d x \\
& +c\left(\int_{0}^{t} \int_{\Omega}|u-v| d x d t\right)^{l} .
\end{aligned}
$$

where $l<1$.

Using Lemma 6, we have

$$
\int_{\Omega}|u(x, s)-v(x, s)| d x \leq c \int_{\Omega}|u(x, \tau)-v(x, \tau)| d x .
$$


Then by the arbitrary of $\tau$,

$$
\int_{\Omega}|u(x, s)-v(x, s)| d x \leq c \int_{\Omega}\left|u_{0}(x)-v_{0}(x)\right| d x
$$

\section{The Uniqueness of Weak Solution}

Lemma 7. Let $u_{t} \in L^{p^{\prime}}\left(0, T ; W^{-1, p^{\prime}}(\Omega)\right)$. For any continuous function $h(s), H(s)=\int_{0}^{s} h(\sigma) d \sigma$, a.e.t. $t_{1}, t_{2} \in(0, T)$, it holds

$$
\int_{t_{1}}^{t_{2}} \int_{\Omega} h(u) u_{t} d x d t=\int_{\Omega}\left(H(u)\left(x, t_{2}\right)-H(u)\left(x, t_{1}\right)\right) d x .
$$

This lemma can be generalized from of Lemma 2.2 in [28] simply; we do not give the details here.

Theorem 8. Let $p_{-}>1, a_{i}(x, t) \in C^{1}\left(\overline{Q_{T}}\right)$ satisfy (8)(9) and $\left(\partial a_{i}(x, t)\right) / \partial t \leq 0, b_{i}(s, x, t)$ be a Lipschitz function on $\mathbb{R} \times \bar{\Omega}$ $\times[0, T]$. If $u(x, t)$ and $v(x, t)$ are two weak solutions of equation (1) with the initial values $u_{0}(x)$ and $v_{0}(x)$, respectively, then, there exists a positive constant $\beta_{j} \geq 2$ such that

$$
\begin{aligned}
& \int_{\Omega}\left(\prod_{j=1}^{N} a_{j}^{\beta_{j}}(x, t)\right)|u(x, t)-v(x, t)|^{2} d x \\
& \quad \leq c \int_{\Omega}\left(\prod_{j=1}^{N} a_{j}^{\beta_{j}}(x, 0)\right)\left|u_{0}(x)-v_{0}(x)\right|^{2} d x .
\end{aligned}
$$

Proof. Let $u(x, t)$ and $v(x, t)$ be two weak solutions of equation (1) with the initial values $u_{0}(x)$ and $v_{0}(x)$, respectively. By a process of limitation, we may choose $\varphi=\chi_{[\tau, s]} \prod_{j=1}^{N} a_{j}^{\beta_{j}}$ $(u-v)$ as a test function. Denoting that $Q_{\tau s}=\Omega \times[\tau, s]$, then,

$$
\begin{aligned}
\iint_{Q_{T s}}(u-v) & \prod_{j=1}^{N} a_{j}^{\beta_{j}} \frac{\partial(u-v)}{\partial t} d x d t \\
=- & \sum_{i=1}^{N} \iint_{Q_{\tau s}} a_{i}(x)\left(\left|u_{x_{i}}\right|^{p_{i}-2} u_{x_{i}}-\left|v_{x_{i}}\right|^{p_{i}-2} v_{x_{i}}\right) \\
\cdot & {\left[(u-v) \prod_{j=1}^{N} a_{j}^{\beta_{j}}\right]_{x_{i}} d x d t-\sum_{i=1}^{N} \iint_{Q_{\tau s}} } \\
\cdot & {\left[b_{i}(u, x, t)-b_{i}(v, x, t)\right]\left[(u-v) \prod_{j=1}^{N} a_{j}^{\beta_{j}}\right]_{x_{i}} d x d t . }
\end{aligned}
$$

At first, we have

$$
\begin{gathered}
\iint_{Q_{\tau s}} a_{i}(x, t)\left(\left|u_{x_{i}}\right|^{p_{i}(x, t)-2} u_{x_{i}}-\left|v_{x_{i}}\right|^{p_{i}(x, t)-2} v_{x_{i}}\right) \\
\cdot(u-v)_{x_{i}} \prod_{j=1}^{N} a_{j}^{\beta_{j}} d x d t \geq 0,
\end{gathered}
$$

$$
\begin{aligned}
& \left|\iint_{Q_{\tau s}}(u-v) a_{i}(x, t)\left(\left|u_{x_{i}}\right|^{p_{i}(x, t)-2} u_{x_{i}}-\left|v_{x_{i}}\right|^{p_{i}(x, t)-2} v_{x_{i}}\right)\left(\prod_{j=1}^{N} a_{j}^{\beta_{j}}\right)_{x_{i}} d x d t\right| \\
& \leq \iint_{Q_{\tau s}}|u-v| a_{i}(x, t)\left(\left|u_{x_{i}}\right|^{p_{i}(x, t)-1}+\left|v_{x_{i}}\right|^{p_{i}(x, t)-1}\right)\left|\left(\prod_{j=1}^{N} a_{j}^{\beta_{j}}\right)_{x_{i}}\right| d x d t \\
& \leq c\left(\int_{\tau}^{s} \int_{\Omega} a_{i}(x, t)\left(\left|u_{x_{i}}\right|^{p_{i}(x, t)}+\left|v_{x_{i}}\right|^{p_{i}(x, t)}\right) d x d t\right)^{1 / p_{1 i}^{\prime}} \\
& \quad \cdot\left(\int_{\tau}^{s} \int_{\Omega} a_{i}(x, t)\left|\left(\prod_{j=1}^{N} a_{j}^{\beta_{j}}\right)_{x_{i} \mid}^{p_{i}(x, t)}\right| u-\left.v\right|^{p_{i}(x, t)} d x d t\right)^{1 / p_{1 i}} \\
& \leq c\left(\int_{\tau}^{s} \int_{\Omega} a_{i}(x)\left(\left.\left|u_{x_{i}}\right|\right|^{p_{i}}+\left|v_{x_{i}}\right|^{p_{i}}\right) d x d t\right)^{\left(p_{i}-1\right) / p_{i}} \\
& \left.\quad \cdot\left(\int_{\tau}^{s} \int_{\Omega} a_{i}(x) \prod_{j=1}^{N}\left|a_{j}^{\beta_{j}-1} a_{j x_{i}}\right|\right)^{p_{i}}|u-v|^{p_{i}} d x d t\right)^{1 / p_{i}} \\
& \leq c\left(\int_{\tau}^{s} \int_{\Omega} a_{i}(x) \prod_{j=1}^{N}\left|a_{j}^{\beta_{j}-1} a_{j x_{i}}\right|^{p_{i}}|u-v|^{p_{i}} d x d t\right)^{1 / p_{i}} .
\end{aligned}
$$

Here, we have used the fact that $\left|a_{x_{i}}\right| \leq c$ and $p_{1 i}=p_{i}^{+}$or $p_{i}^{-}$according to

$$
\int_{\tau}^{s} \int_{\Omega} a_{i}(x, t)\left|\left(\prod_{j=1}^{N} a_{j}^{\beta_{j}}\right)_{x_{i}}\right|_{i}^{p_{i}(x, t)}|u-v|^{p_{i}(x, t)} d x d t \leq 1,
$$

or

$$
\int_{\tau}^{s} \int_{\Omega} a_{i}(x, t)\left|\left(\prod_{j=1}^{N} a_{j}^{\beta_{j}}\right)_{x_{i}}\right|^{p_{i}(x, t)}|u-v|^{p_{i}(x, t)} d x d t>1 .
$$

$p^{\prime}{ }_{1 i}$ has a similar meaning. Now, if we denote that $Q_{1}$ $=\left\{(x, t) \in Q_{T}: 1<p(x, t)<2\right\}$ and $Q_{2}=\left\{(x, t) \in Q_{T}: p(x\right.$, $t) \geq 2\}$, by that $\beta_{i} \geq 2$, we have

$$
\begin{aligned}
& \left(\iint_{Q_{\tau i} \bigcap Q_{2}} a_{i}(x, t) \prod_{j=1}^{N}\left|a_{j}^{\beta_{j}-1} a_{j x_{i}}\right|^{p_{i}(x, t)}|u-v|^{p_{i}(x, t)} d x d t\right)^{1 / p_{1 i}} \\
& \quad \leq c\left(\int_{\tau}^{s} \int_{\Omega} \prod_{j=1}^{N} a_{j}^{\beta_{j}}|u-v|^{2} d x d t\right)^{1 / p_{1 i}} .
\end{aligned}
$$

and by the Hölder inequality,

$$
\begin{aligned}
& \left(\iint_{Q_{\tau s} \cap Q_{1}} a_{i}(x, t) \prod_{j=1}^{N}\left|a_{j}^{\beta_{j}-1} a_{j x_{i}}\right|^{p_{i}(x, t)}|u-v|^{p_{i}(x, t)} d x d t\right)^{1 / p_{1 i}} \\
& \quad \leq c\left(\int_{\tau}^{s} \int_{\Omega} \prod_{j=1}^{N} a_{j}^{\beta_{j}}|u-v|^{2} d x d t\right)^{1 / k} .
\end{aligned}
$$

where $k<1$. 
Combing (67)-(71), we obtain

$$
\begin{aligned}
& \left|\iint_{Q_{\tau s}}(u-v) a_{i}(x, t)\left(\left|u_{x_{i}}\right|^{p_{i}(x, t)-2} u_{x_{i}}-\left|v_{x_{i}}\right|^{p_{i}(x, t)-2} v_{x_{i}}\right)\left(\prod_{j=1}^{N} a_{j}^{\beta_{j}}\right)_{x_{i}} d x d t\right| \\
& \quad \leq c\left(\int_{\tau}^{s} \int_{\Omega} \prod_{j=1}^{N} a_{j}^{\beta_{j}}|u-v|^{2} d x d t\right)^{l}
\end{aligned}
$$

where $l<1$.

Secondly,

$$
\begin{aligned}
& \iint_{Q_{\tau s}}\left[b_{i}(u, x, t)-b_{i}(v, x, t)\right]\left[(u-v) \prod_{j=1}^{N} a_{j}^{\beta_{j}}\right]_{x_{i}} d x d t \\
& =\iint_{Q_{\tau s}}\left[b_{i}(u, x, t)-b_{i}(v, x, t)\right](u-v)\left(\prod_{j=1}^{N} a_{j}^{\beta_{j}}\right)_{x_{i}} d x d t \\
& \quad+\iint_{Q_{s}}\left[b_{i}(u, x, t)-b_{i}(v, x, t)\right](u-v)_{x_{i}} \prod_{j=1}^{N} a_{j}^{\beta_{j}} d x d t .
\end{aligned}
$$

For the first term on the right hand side of (73), by that $\beta_{j} \geq 2,\left|a_{j x_{i}}\right| \leq c$, using the Hölder inequality, we have

$$
\begin{gathered}
\iint_{Q_{\tau s}}\left[b_{i}(u, x, t)-b_{i}(v, x, t)\right](u-v)\left(\prod_{j=1}^{N} a_{j}^{\beta_{j}}\right)_{x_{i}} d x d t \\
=\int_{\tau}^{s} \int_{\Omega}\left[b_{i}(u, x, t)-b_{i}(v, x, t)\right] \\
\cdot(u-v) \sum_{k=1}^{N}\left(\beta_{k} a_{k}^{\beta_{k}-1} a_{k x_{i}} \prod_{j=1, j \neq k}^{N} a_{j}^{\beta_{j}}\right) d x d t \\
\leq c \int_{\tau}^{s} \int_{\Omega}|u-v| \sum_{k=1}^{N}\left(\beta_{k} a_{k}^{\beta_{k}-1} a_{k x_{i}} \prod_{j=1, j \neq k}^{N} a_{j}^{\beta_{j}}\right) d x d t \\
\leq c\left(\int_{\tau}^{s} \int_{\Omega} \prod_{j=1}^{N} a_{j}^{\beta_{j}}|u-v|^{2} d x d t\right)^{1 / 2} \cdot
\end{gathered}
$$

For the second term on the right hand side of (73), since $\beta_{i} \geq 2$, denoting $p_{i}^{\prime}(x, t)=p_{i}(x, t) /\left(p_{i}(x, t)-1\right)$ as usual, we have

$$
\left(\beta_{i}-\frac{1}{p_{i}(x, t)}\right) p_{i}^{\prime}(x, t) \geq \beta_{i}
$$

By this inequality, we deduce

$$
\begin{aligned}
& \left|\iint_{Q_{t s}}\left[b_{i}(u, x, t)-b_{i}(v, x, t)\right](u-v)_{x_{i}} \prod_{j=1}^{N} a_{j}^{\beta_{j}} d x d t\right| \\
& \leq c\left(\int_{\tau}^{s} \int_{\Omega} a_{i}^{\left(\beta_{i}-1 / p_{i}(x, t)\right) p_{i}^{\prime}(x, t)}\left(\prod_{j=1, j \neq i}^{N} a_{j}^{\beta_{j}}\left|b_{i}(u, x, t)-b_{i}(v, x, t)\right|\right)^{p_{i}(x, t)} d x d t\right)^{1 / p_{1 i^{\prime}}} \\
& \cdot\left(\int_{\tau}^{s} \int_{\Omega} a_{i}(x, t)\left(\left|u_{x_{i}}\right|^{p_{i}(x, t)}+\left|v_{x_{i}}\right|^{p_{i}(x, t)}\right) d x d t\right)^{1 / p_{1 i}} \\
& \leq c\left(\int_{\tau}^{s} \int_{\Omega} a_{i}^{\left(\beta_{i}-1 \mid p_{i}(x, t)\right) p_{i}^{\prime}(x, t)}\left(\prod_{j=1, j \neq i}^{N} a_{j}^{\beta_{j}}\left|b_{i}(u, x, t)-b_{i}(v, x, t)\right|\right)^{p_{i}(x, t)} d x d t\right)^{1 / p_{11}{ }^{\prime}} \\
& \leq c\left(\int_{\tau}^{s} \int_{\Omega} \prod_{j=1}^{N} a_{j}^{\beta_{j}}|u-v|^{p_{i}(x, t)} d x d t\right)^{1 / p_{11}^{\prime}{ }^{\prime}} .
\end{aligned}
$$

Defining $Q_{1}, Q_{2}$ as above, if $p_{i}(x, t) \geq 2$, then $1<p_{i}^{\prime}(x, t)$ $\leq 2$. By the Hölder inequality,

$$
\begin{gathered}
\left(\iint_{Q_{\tau s} \cap Q_{2}} \prod_{j=1}^{N} a_{j}^{\beta_{j}}|u-v|^{p_{i}(x, t)} d x d t\right)^{1 / p_{1 i}{ }^{\prime}} \\
\quad \leq c\left(\int_{\tau}^{s} \int_{\Omega} \prod_{j=1}^{N} a_{j}^{\beta_{j}}|u-v|^{2} d x d t\right)^{1 / k},
\end{gathered}
$$

where $k<1$.

$$
\begin{aligned}
& \text { If } 1<p_{i}(x, t)<2 \text {, then } p_{i}^{\prime}(x, t)>2, \\
& \left(\iint_{Q_{\tau s} \bigcap Q_{1}} \int_{\Omega} \prod_{j=1}^{N} a_{j}^{\beta_{j}}|u-v|^{p_{i}{ }^{\prime}(x, t)} d x d t\right)^{1 / p_{1 i}{ }^{\prime}} \\
& \quad \leq c\left(\int_{\tau}^{s} \int_{\Omega} \prod_{j=1}^{N} a_{j}^{\beta_{j}}|u-v|^{2} d x d t\right)^{1 / p_{1 i}{ }^{\prime}}
\end{aligned}
$$

From (76)-(78), we obtain

$$
\begin{aligned}
& \left|\iint_{Q_{\tau s}}\left[b_{i}(u, x, t)-b_{i}(v, x, t)\right](u-v)_{x_{i}} \prod_{j=1}^{N} a_{j}^{\beta_{j}} d x d t\right| \\
& \quad \leq c\left(\int_{\tau}^{s} \int_{\Omega} \prod_{j=1}^{N} a_{j}^{\beta_{j}}|u-v|^{2} d x d t\right)^{l},
\end{aligned}
$$

where $l<1$. 
Once more, by Lemma 7, we have

$$
\begin{aligned}
& \iint_{Q_{\tau s}}(u-v) \prod_{j=1}^{N} a_{j}^{\beta_{j}} \frac{\partial(u-v)}{\partial t} d x d t \\
& =\iint_{Q_{\tau s}}(u-v) \sqrt{\prod_{j=1}^{N} a_{j}^{\beta_{j}}} \frac{\partial\left[\sqrt{\prod_{j=1}^{N} a_{j}^{\beta_{j}}}(u-v)\right]}{\partial t} d x d t \\
& \quad-\iint_{Q_{\tau s}}(u-v)^{2} \sqrt{\prod_{j=1}^{N} a_{j}^{\beta_{j}}} \frac{\partial \sqrt{\prod_{j=1}^{N} a_{j}^{\beta_{j}}}}{\partial t} d x d t \\
& =\int_{\Omega} \prod_{j=1}^{N} a_{j}^{\beta_{j}}[u(x, s)-v(x, s)]^{2} d x \\
& \quad-\int_{\Omega} \prod_{j=1}^{N} a_{j}^{\beta_{j}}[u(x, \tau)-v(x, \tau)]^{2} d x \\
& \quad-\iint_{Q_{\tau s}}(u-v)^{2} \sqrt{\prod_{j=1}^{N} a_{j}^{\beta_{j}}} \frac{\partial \sqrt{\prod_{j=1}^{N} a_{j}^{\beta_{j}}}}{\partial t} d x d t .
\end{aligned}
$$

According to (65), (66), (72), (74), (79), and (80), since $\left(\partial a_{i}(x, t)\right) / \partial t \leq 0$ and $\beta_{j} \geq 2$, we have

$$
\begin{gathered}
\int_{\Omega} \prod_{j=1}^{N} a_{j}^{\beta_{j}}[u(x, s)-v(x, s)]^{2} d x-\int_{\Omega} \prod_{j=1}^{N} a_{j}^{\beta_{j}}[u(x, \tau)-v(x, \tau)]^{2} d x \\
\leq c\left(\int_{\tau}^{s} \int_{\Omega} \prod_{j=1}^{N} a_{j}^{\beta_{j}}|u(x, t)-v(x, t)|^{2} d x d t\right)^{l} \\
\quad+\iint_{Q_{\tau s}}(u-v)^{2} \sqrt{\prod_{j=1}^{N} a_{j}^{\beta_{j}}} \frac{\partial \sqrt{\prod_{j=1}^{N} a_{j}^{\beta_{j}}}}{\partial t} d x d t \\
\leq c\left(\int_{\tau}^{s} \int_{\Omega} \prod_{j=1}^{N} a_{j}^{\beta_{j}}|u(x, t)-v(x, t)|^{2} d x d t\right)^{l},
\end{gathered}
$$

where $l<1$. By (81), using the generalized Gronwall inequality, we deduce

$$
\begin{aligned}
& \int_{\Omega} \prod_{j=1}^{N} a_{j}(x, s)^{\beta_{j}}|u(x, s)-v(x, s)|^{2} d x \\
& \quad \leq \int_{\Omega} \prod_{j=1}^{N} a_{j}(x, \tau)^{\beta_{j}}|u(x, \tau)-v(x, \tau)|^{2} d x .
\end{aligned}
$$

Thus, by the arbitrary of $\tau$, we have

$$
\begin{aligned}
& \int_{\Omega} \prod_{j=1}^{N} a_{j}(x, s)^{\beta_{j}}|u(x, s)-v(x, s)|^{2} d x \\
& \quad \leq \int_{\Omega} \prod_{j=1}^{N} a_{j}(x, 0)^{\beta_{j}}\left|u_{0}(x)-v_{0}(x)\right|^{2} d x .
\end{aligned}
$$

By this theorem, Theorem 5 is true clearly.

\section{The Strong Solutions Dependent on the Initial Value}

For the completeness of the paper, we will give a basic theorem about the existence of strong solutions.

Theorem 9. If $p_{-}>2, a_{i}(x, t) \in C(\bar{\Omega})$ satisfies (8) and (9), $b_{i}$ $(s, x, t)$ is a $C^{l}$ function on $\mathbb{R} \times \bar{\Omega} \times[0, T]$,

$$
\begin{aligned}
& u_{0} \in L^{\infty}(\Omega),\left|u_{0 x_{i}}\right| \in L^{p_{i}}(\Omega), \quad i=1,2, \cdots, N, \\
& \int_{\Omega} a_{i}(x, t)^{-1 /\left(p_{i}(x, t)-1\right)} d x<\infty, \quad i=1,2, \cdots, N, \\
&\left|b_{i}(s, x, t)\right| \leq c a_{i}(x, t)^{1 / p_{i}(x, t)},\left|b_{i s}(s, x, t)\right| \\
& \leq c a_{i}(x, t)^{1 / p_{i}(x, t)}, \quad i=1,2, \cdots, N
\end{aligned}
$$

then equation (1) with initial value (2) has a weak solution.

Here, $b_{i s}(s, x, t)=\left(\partial b_{i}(s, x, t)\right) / \partial s$. Before we give the proof of Theorem 9, we would like to point out that condition (86) is just a sufficient condition; we also can use other conditions to replace them. For example, when $a_{i}(x, t) \equiv a(x), p_{i}(x, t)=p_{i}$, by the conditions

$$
\begin{aligned}
& \int_{\Omega} a_{i}(x)^{-2 /\left(p_{i}-2\right)} d x<c, \\
& \left|b_{i s}(s, x, t)\right| \leq c a(x)^{1 / p_{i}} .
\end{aligned}
$$

Theorem 9 had been obtained in [19].

Proof of Theorem 9. Consider the following regularized problem

$$
\begin{aligned}
u_{\varepsilon t} & -\sum_{i=1}^{N} \frac{\partial}{\partial x_{i}}\left(\left(a_{i}(x, t)+\varepsilon\right)\left|u_{\varepsilon x_{i}}\right|^{p_{i}(x, t)-2} u_{\varepsilon x_{i}}\right) \\
& -\sum_{i=1}^{N} \frac{\partial b_{i}\left(u_{\varepsilon}, x, t\right)}{\partial x_{i}}=0, \quad(x, t) \in Q_{T}, \\
u_{\varepsilon}(x, t) & =0, \quad(x, t) \in \partial \Omega \times(0, T), \\
u_{\varepsilon}(x, 0) & =u_{\varepsilon 0}(x), \quad x \in \Omega .
\end{aligned}
$$

Here, $u_{\varepsilon 0} \in C_{0}^{\infty}(\Omega), \quad\left|u_{\varepsilon 0}\right|_{L^{\infty}(\Omega)} \leq\left|u_{0}\right|_{L^{\infty}(\Omega)}$, and $\left|\nabla u_{\varepsilon 0}\right|$ converges to $\left|\nabla u_{0}(x)\right|$ in $L^{p_{+}}(\Omega)$. It is well-known that the above problem has a unique weak solution $u_{\varepsilon} \in L^{\infty}\left(Q_{T}\right)$ and $a_{i}(x, t)\left|u_{x_{i}}\right|^{p_{i}(x, t)} \in L^{1}\left(Q_{\mathrm{T}}\right)[8]$, and

$$
\left\|u_{\varepsilon}\right\|_{L^{\infty}\left(Q_{T}\right)} \leq c(M)
$$

where $M=\left\|u_{0}(x)\right\|_{L^{\infty}(\Omega)}$. 
Multiplying (88) by $u_{\varepsilon}$ and integrating it over $Q_{T}$, then

$$
\begin{gathered}
\frac{1}{2} \int_{\Omega} u_{\varepsilon}^{2} d x+\sum_{i=1}^{N} \iint_{Q_{T}}\left(a_{i}(x, t)+\varepsilon\right)\left|u_{\varepsilon x_{i}}\right|^{p_{i}(x, t)} d x d t \\
\quad+\sum_{i=1}^{N} \iint_{Q_{T}} \frac{\partial b_{i}\left(u_{\varepsilon}, x, t\right)}{\partial x_{i}} u_{\varepsilon} d x d t=\frac{1}{2} \int_{\Omega} u_{\varepsilon 0}^{2} d x .
\end{gathered}
$$

If $\left|b_{i}\left(u_{\varepsilon}, x, t\right)\right| \leq c a_{i}(x, t)^{1 /\left(p_{i}(x, t)\right)}$, by that $\int_{\Omega} a_{i}$ $(x, t)^{-1 /\left(p_{i}(x, t)-1\right)} d x<\infty$, then

$$
\begin{aligned}
\left|\int_{\Omega} \frac{\partial b_{i}\left(u_{\varepsilon}, x, t\right)}{\partial x_{i}} u_{\varepsilon} d x\right| & =\left|-\int_{\Omega} \frac{\partial u_{\varepsilon}}{\partial \mathrm{x}_{i}} b_{i}\left(u_{\varepsilon}, x, t\right) d x\right| \\
& \leq c \int_{\Omega} a_{i}(x, t)^{1 / p_{i}(x, t)}\left|\frac{\partial u_{\varepsilon}}{\partial x_{i}}\right| d x \\
& \leq c \int_{\Omega} a_{i}(x, t)\left|u_{x_{i}}\right|^{p_{i}(x, t)} d x+c .
\end{aligned}
$$

Accordingly, by (92), we have

$$
\int_{\Omega} u_{\varepsilon}^{2} d x+\sum_{i=1}^{N} \iint_{Q_{T}}\left(a_{i}(x, t)+\varepsilon\right)\left|u_{\varepsilon x_{i}}\right|^{p_{i}(x, t)} d x d t \leq c .
$$

Multiplying (88) by $u_{\varepsilon t}$, integrating it over $Q_{T}$, then it yields

$$
\begin{aligned}
\iint_{Q_{T}}\left|u_{\varepsilon t}\right|^{2} d x d t= & \sum_{i=1}^{N} \iint_{Q_{T}} \frac{\partial}{\partial x_{i}}\left(\left(a_{i}(x, t)+\varepsilon\right)\left|u_{\varepsilon x_{i}}\right|^{p_{i}(x, t)-2} u_{\varepsilon x_{i}}\right) u_{\varepsilon t} d x d t \\
& +\sum_{i=1}^{N} \iint_{Q_{T}} u_{\varepsilon t} \frac{\partial b_{i}\left(u_{\varepsilon}, x, t\right)}{\partial x_{i}} d x d t .
\end{aligned}
$$

Note that $p_{-}>2$,

$$
\begin{aligned}
-\left(\left|u_{\varepsilon x_{i}}\right|^{2}+\varepsilon\right)^{\left(p_{i}(x, t)-2\right) / 2} u_{\varepsilon x_{i}} \cdot \frac{\partial}{\partial x_{i}} u_{\varepsilon t} \\
=-\frac{1}{2} \frac{\partial}{\partial t} \int_{0}^{\left.u_{\varepsilon x_{i}}\right|^{2}+\varepsilon} s^{\left(p_{i}(x, t)-2\right) / 2} d s \\
+\frac{1}{2} \int_{0}^{\left|u_{\varepsilon x_{i}}\right|^{2}+\varepsilon} \frac{\partial}{\partial t} s^{\left(p_{i}(x, t)-2\right) / 2} d s \\
=-\frac{1}{2} \frac{\partial}{\partial t} \int_{0}^{\left|u_{\varepsilon x_{i}}\right|^{2}+\varepsilon} s^{\left(p_{i}(x, t)-2\right) / 2} d s \\
+\frac{1}{4} \int_{0}^{\left|u_{\varepsilon x_{i}}\right|^{2}+\varepsilon} s^{\left(p_{i}(x, t)-2\right) / 2} \ln s \frac{\partial p_{i}(x, t)}{\partial t} d s \\
=-\frac{1}{2} \frac{\partial}{\partial t} \int_{0}^{\left|u_{\varepsilon x_{i}}\right|^{2}+\varepsilon} s^{\left(p_{i}(x, t)-2\right) / 2} d s \\
\quad+\frac{1}{4} \int_{0}^{\left|u_{\varepsilon x_{i}}\right|^{2}+\varepsilon} s^{\left(p_{i}(x, t)-2\right) / 2} \ln s \frac{\partial p_{i}(x, t)}{\partial t} d s,
\end{aligned}
$$

and by the Young inequality,

$$
\begin{aligned}
& \left|\iint_{Q_{T}}\left(a_{i}(x, t)+\varepsilon\right) \int_{0}^{\left|u_{\varepsilon x_{i}}\right|^{2}+\varepsilon} s^{\left(p_{i}(x, t)-2\right) / 2} \ln s \frac{\partial p_{i}(x, t)}{\partial t} d s d x d t\right| \\
& \quad \leq c \iint_{Q_{T}}\left(a_{i}(x, t)+\varepsilon\right)\left(\left|u_{\varepsilon x_{i}}\right|^{2}+\varepsilon\right) d x d t \\
& \quad \leq c \iint_{Q_{T}}\left(a_{i}(x, t)+\varepsilon\right)\left(\left|u_{\varepsilon x_{i}}\right|^{p_{i}(x, t)}+1\right) d x d t \leq c .
\end{aligned}
$$

We have

$$
\begin{aligned}
\sum_{i=1}^{N} & \iint_{Q_{T}} \frac{\partial}{\partial x_{i}}\left(\left(a_{i}(x, t)+\varepsilon\right)\left(\left|u_{\varepsilon x_{i}}\right|^{2}+\varepsilon\right)^{\left(p_{i}(x, t)-2\right) / 2} u_{\varepsilon x_{i}}\right) u_{\varepsilon t} d x d t \\
= & -\sum_{i=1}^{N} \iint_{Q_{T}}\left(a_{i}(x, t)+\varepsilon\right)\left(\left|u_{\varepsilon x_{i}}\right|^{2}+\varepsilon\right)^{\left(p_{i}(x, t)-2\right) / 2} u_{\varepsilon x_{i}} \\
& \cdot \frac{\partial}{\partial x_{i}} u_{\varepsilon t} d x d t=-\sum_{i=1}^{N} \frac{1}{2} \iint_{Q_{T}}\left(a_{i}(x, t)+\varepsilon\right) \frac{d}{d t} \\
& \int_{0}^{\left|u_{\varepsilon x_{i}}\right|^{2}+\varepsilon} s^{\left(p_{i}(x, t)-2\right) / 2} d s d x d t+\frac{1}{4} \sum_{i=1}^{N} \iint_{Q_{T}}\left(a_{i}(x, t)+\varepsilon\right) \\
& \int_{0}^{\left|u_{\varepsilon x_{i}}(x, t)\right|^{2}+\varepsilon} s^{\left(p_{i}(x, t)-2\right) / 2} \ln s \frac{\partial p_{i}(x, t)}{\partial t} d s d x d t .
\end{aligned}
$$

$$
\begin{aligned}
& \text { If }\left|b_{i u}\left(u_{\varepsilon}, x, t\right)\right| \leq c a_{i}(x, t)^{1 / p_{i}(x, t)}, \\
& \qquad \begin{aligned}
\iint_{Q_{T}} & u_{\varepsilon t} \frac{\partial b_{i}\left(u_{\varepsilon}, x, t\right)}{\partial x_{i}} d x d t \\
\leq & \iint_{Q_{T}}\left|b_{i u}\left(u_{\varepsilon}, x, t\right)\right|\left|u_{\varepsilon x_{i}}\right|\left|u_{\varepsilon t}\right| d x d t \\
& \quad+\iint_{Q_{T}}\left|b_{i x_{i}}\left(u_{\varepsilon}, x, t\right)\right|\left|u_{\varepsilon t}\right| d x d t \\
\leq & \frac{1}{2} \iint_{Q_{T}}\left|u_{\varepsilon t}\right|^{2} d x d t+c \iint_{Q_{T}} a_{i}(x, t) \\
& \cdot\left|u_{\varepsilon x_{i}}\right|^{p_{i}(x, t)} d x d t+c .
\end{aligned}
\end{aligned}
$$

Combining (95), (98), and (99), we have

$$
\iint_{Q_{T}}\left|u_{\varepsilon t}\right|^{2} d x d t+\sum_{i=1}^{N} \iint_{Q_{T}}\left(a_{i}(x, t)+\varepsilon\right) \frac{d}{d t} \int_{0}^{\left|u_{\varepsilon x_{i}}\right|^{2}} s^{\left(p_{i}(x, t)-2\right) / 2} d s d x d t \leq c .
$$

By the above inequality, we have

$$
\iint_{Q_{T}}\left|u_{\varepsilon t}\right|^{2} d x d t \leq c+c \sum_{i=1}^{N} \int_{\Omega}\left(a_{i}(x, 0)+\varepsilon\right)\left|u_{\varepsilon 0 x_{i}}\right|^{p_{i}} d x \leq c .
$$

Thus, by (91), (94), and (101), there exist a function $u$ and a $n$-dimensional vector function $\overleftarrow{\zeta}=\left(\zeta_{1}, \cdots, \zeta_{n}\right)$ satisfying that $u_{\varepsilon} \rightarrow u$ a.e. in $Q_{T}$, and 


$$
\begin{aligned}
u \in L^{\infty}\left(Q_{T}\right),\left|\zeta_{i}\right| & \in L^{1}\left(0, T ; L^{p_{i}(x, t) /\left(p_{i}(x, t)-1\right)}(\Omega)\right), \\
u_{\varepsilon} & \rightarrow * u, \operatorname{in} L^{\infty}\left(Q_{T}\right), \\
b_{i}\left(u_{\varepsilon}, x, t\right) & \rightarrow b_{i}(u, x, t), \text { a.e.in } Q_{T}, \\
a_{i}(x, t)\left|u_{\varepsilon x_{i}}\right|^{p_{i}(x, t)-2} u_{\varepsilon x_{i}} & \rightarrow \zeta_{i}, \operatorname{in} L^{1}\left(0, T ; L^{p_{i}(x, t) /\left(p_{i}(x, t)-1\right)}(\Omega)\right) .
\end{aligned}
$$

Similar as with Theorem 2.1 of Chapter 2 in [1] (also, one can refer to [19] in which $p_{i}(x, t)=p_{i}$ is just a constant), we are able to show that

$$
\begin{aligned}
\lim _{\varepsilon \rightarrow 0} & \sum_{i=1}^{N} \iint_{Q_{T}}\left(a_{i}(x, t)+\varepsilon\right)\left|u_{\varepsilon x_{i}}\right|^{p_{i}(x, t)-2} u_{\varepsilon x_{i}} \varphi_{x_{i}} d x d t \\
= & \lim _{\varepsilon \rightarrow 0} \sum_{i=1}^{N} \iint_{Q_{T}} a_{i}(x, t)\left|u_{\varepsilon x_{i}}\right|^{p_{i}(x, t)-2} u_{\varepsilon x_{i}} \varphi_{x_{i}} d x d t \\
= & \iint_{Q_{T}} \overleftarrow{\zeta} \cdot \nabla \varphi d x d t=\sum_{i=1}^{N} \iint_{Q_{T}} a_{i}(x, t) \\
& \cdot\left|u_{x_{i}}\right|^{p_{i}(x, t)-2} u_{x_{i}} \varphi_{x_{i}} d x d t,
\end{aligned}
$$

for any $\varphi \in C_{0}^{1}\left(Q_{T}\right)$. At last, by a process of limit, we can choose the test function $\varphi(x, t)=\chi_{\left[t_{1}, t_{2}\right]} \phi(x)$ in which $\phi(x)$ $\in C_{0}^{\infty}(\Omega)$ and $\chi_{\left[t_{1}, t_{2}\right]}$ is the characteristic function of $\left[t_{1}, t_{2}\right]$ $\subset(0, T)$. Then,

$$
\begin{aligned}
& \sum_{i=1}^{N} \int_{t_{1}}^{t_{2}} \int_{\Omega}\left[a_{i}(x, t)\left|u_{x_{i}}\right|^{p_{i}(x, t)-2} u_{x_{i}} \phi_{x_{i}}+b_{i}(u, x, t) \phi_{x_{i}}\right] d x d t \\
& \quad=\int_{\Omega}\left(u\left(x, t_{2}\right)-u\left(x, t_{1}\right)\right) \phi(x) d x
\end{aligned}
$$

Let $t=t_{2}$ and $t_{1} \rightarrow 0$. Then, we have (23) and $u$ is a strong solution of equation (1) with the initial value (2) in the sense of Definition 2.

At last, we give a simple comment. The condition $a_{i} u_{x_{i}}$ $\in L^{1}(\Omega)$ can not assure the boundary value condition

$$
u(x, t)=0, \quad(x, t) \in \Sigma_{p} \times(0, T) \subseteq \partial \Omega \times(0, T)
$$

is imposed in the sense of the trace. In fact, we have the following proposition.

Proposition 10. If $a_{i}(x, t)$ satisfies (85), i.e.,

$$
\int_{\Omega} a_{i}(x, t)^{-1 /\left(p_{i}(x, t)-1\right)} d x<\infty, \quad i=1,2, \cdots, N
$$

Then,

$$
\int_{\Omega}\left|u_{x_{i}}\right| d x \leq c(T)<\infty, \quad i=1,2, \cdots, N
$$

Thus, if $a_{i}(x, t)$ satisfies (85), the partial boundary value condition (105) can be imposed in the sense of trace. However, in this paper, we pay our attention on the studying the stability (or the uniqueness) of solutions independent of the boundary value condition (105), so Proposition 10 is not important.

\section{Conclusion}

It is clear of that Lemma 6 has a wider applications than the classical Gronwall inequality. Moreover, compared with reference [18], there is at least the essential difference in two aspects. The first one is that condition (9), i.e.,

$$
\prod_{i=1}^{N} a_{i}(x, t)=0,(x, t) \in \partial \Omega \times[0, T]
$$

is much weaker than condition (5) appearing in [19], i.e.

$$
a_{i}(x)=0, \quad x \in \partial \Omega, i=1,2, \cdots, N
$$

Such a degeneracy is the special nature of the anisotropic equation. The second one is that we have not used boundary value condition (3) throughout this paper; in other words, condition (9) may replace boundary value condition (3) in some way. Moreover, using some techniques developed by the second author in his work [10], in which the wellposedness of weak solutions to equation,

$$
\begin{aligned}
\left(|v|^{\beta-1} v\right)_{t}= & \operatorname{div}\left(b(x, t)|\nabla v|^{p(x, t)-2} \nabla v\right) \\
& +\sum_{i=1}^{N} g_{i}(x, t) \frac{\partial \gamma_{i}(v)}{\partial x_{i}}, \quad(x, t) \in Q_{T}
\end{aligned}
$$

has been discussed; the method used in this paper can be applied to study a more general equation

$$
\begin{aligned}
\left(|u|^{\beta-1} u\right)_{t}= & \sum_{i=1}^{N} \frac{\partial}{\partial x_{i}}\left(a_{i}(x, t)\left|u_{x_{i}}\right|^{p_{i}(x, t)-2} u_{x_{i}}\right) \\
& +f(x, t, u, \nabla u), \quad(x, t) \in Q_{T}
\end{aligned}
$$

in the future.

\section{Data Availability}

There is not any data in the paper.

\section{Conflicts of Interest}

The authors declare that they have no competing interests.

\section{Authors' Contributions}

The authors read and approve the final manuscript. 


\section{Acknowledgments}

The paper is supported by the Natural Science Foundation of Fujian Province of China (no. 2019J01858). The authors would like to thank everyone for their help.

\section{References}

[1] S. V. Antontsev, J. I. Diaz, and S. Shmarev, Energy Methods for Free Boundary Problems: Applications to Nonlinear PDEs and Fluid Mechanics, vol. 48 of Progress in Nonlinear Differential Equations and Their Applications, Birkhäuser Boston, Inc, Boston, MA, USA, 2002.

[2] E. DiBenedetto, Degenerate Parabolic Equations, Spring-Verlag, New York, NY, USA, 1993.

[3] Z. Wu, J. Zhao, J. Yin, and H. Li, Nonlinear Diffusion Equations, Word Scientific Publishing, Singapore, 2001.

[4] S. V. Antontsev and S. Shmarev, "Existence and uniqueness for doubly nonlinear parabolic equations with nonstandard growth conditions," Differential Equations and Applications, vol. 4, no. 1, pp. 67-94, 2009.

[5] H. Zhan, "Infiltration equation with degeneracy on the boundary," Acta Applicandae Mathematicae, vol. 153, no. 1, pp. 147161, 2018.

[6] M. Ruzicka, Electrorheological Fluids: Modeling and Mathematical Theory, vol. 1748 of Lecture Notes in Mathematics, Springer, Berlin, 2000.

[7] E. Acerbi and G. Mingione, "Regularity results for stationary electro-rheological fluids," Archive for Rational Mechanics and Analysis, vol. 164, no. 3, pp. 213-259, 2002.

[8] S. Antontsev and S. Shmarev, "Anisotropic parabolic equations with variable nonlinearity," Publicacions Matemàtiques, vol. 53, pp. 355-399, 2009.

[9] S. Antontsev and S. Shmarev, "Parabolic equations with double variable nonlinearities," Mathematics and Computers in Simulation, vol. 81, no. 10, pp. 2018-2032, 2011.

[10] H. Zhan, "The nonnegative weak solution of a degenerate parabolic equation with variable exponent growth order," Boundary Value Problems, vol. 2020, no. 1, 2020.

[11] S. Lian, W. Gao, H. Yuan, and C. Cao, "Existence of solutions to an initial Dirichlet problem of evolutional $\mathrm{p}(\mathrm{x})$-Laplace equations," Annales de l'Institut Henri Poincare (C) Non Linear Analysis, vol. 29, no. 3, pp. 377-399, 2012.

[12] A. S. Tersenov, "The one dimensional Parabolic $\mathrm{p}(\mathrm{x})$-Laplace equation," Nonlinear Differential Equations and Applications NoDEA, vol. 23, no. 3, pp. 1-11, 2016.

[13] A. S. Tersenov and A. S. Tersenov, "Existence of Lipschitz continuous solutions to the Cauchy-Dirichlet problem for anisotropic parabolic equations," Journal of Functional Analysis, vol. 272, no. 10, pp. 3965-3986, 2017.

[14] J. Aramaki, "Hölder continuity with exponent $(1+\alpha) / 2$ in the time variable for solutions of parabolic equations," Electronic Journal of Differential Equations, vol. 96, pp. 1-7, 2015.

[15] H. Zhan, "The stability of the anisotropic parabolic equation with the variable exponent," Boundary Value Problems, vol. 2017, no. 1, 2017.

[16] H. Zhan and J. Wen, "Evolutionary $\mathrm{p}(\mathrm{x})$-Laplacian equation free from the limitation of the boundary value," Electronic Journal of Differential Equations, vol. 143, pp. 1-13, 2016.
[17] H. Zhan and B. Xu, "A new kind of weak solution of nonNewtonian fluid equation," Journal of Function Spaces, vol. 2017, 8 pages, 2017.

[18] H. Zhan and Z. Feng, "Solutions of evolutionary $p(x)$-Laplacian equation based on the weighted variable exponent space," Zeitschrift für Angewandte Mathematik und Physik, vol. 68, no. 6, 2017.

[19] H. Zhan, "On anisotropic parabolic equations with a nonlinear convection term depending on the spatial variable," Advances in Difference Equations, vol. 2019, no. 1, 2019.

[20] H. Zhan and Z. Feng, "Well-posedness problem of an anisotropic parabolic equation," Journal of Differential Equations, vol. 268, no. 2, pp. 389-413, 2020.

[21] H. Zhan and Z. Feng, "Solutions of evolutionary equation based on the anisotropic variable exponent Sobolev space," Zeitschrift für Angewandte Mathematik und Physik, vol. 70, no. 4, 2019.

[22] B. Liu and M. Dong, "A nonlinear diffusion problem with convection and anisotropic nonstandard growth conditions," Nonlinear Analysis: Real World Applications, vol. 48, pp. 383-409, 2019.

[23] P. Marcellini, “A variational approach to parabolic equations under general and p,q-growth conditions," Nonlinear Analysis, vol. 194, p. 111456, 2020.

[24] S. Zeng, L. Gasiński, P. Winkert, and Y. Bai, "Existence of solutions for double phase obstacle problems with multivalued convection term," Journal of Mathematical Analysis and Applications, no. article 123997, 2020.

[25] V. V. Zhikov, "On the density of smooth functions in SobolevOrlicz spaces," Journal of Mathematical Sciences, vol. 132, no. 3, pp. 285-294, 2006.

[26] X. Fan and D. Zhao, "On the Spaces $L^{p(x)}(\Omega)$ and $W^{m, p(x)}$," Journal of Mathematical Analysis and Applications, vol. 263, no. 2, pp. 424-446, 2001.

[27] O. Kovacik and J. Rakosnik, "On spaces $L^{p(x)}$ and $W^{k, p(x), "}$ Czechoslovak Mathematical Journal, vol. 41, no. 4, pp. 592618, 1991.

[28] K. Ho and I. Sim, "On degenerate $\mathrm{p}(\mathrm{x})$-Laplace equations involving critical growth with two parameters," Nonlinear Analysis, vol. 132, pp. 95-114, 2016. 